\title{
An Attempt to Avoid Exact Jacobian and Nonlinear Equations in the Numerical Solution of Stiff Differential Equations
}

\author{
By Trond Steihaug and Arne Wolfbrandt
}

\begin{abstract}
A class of linear implicit methods for numerical solution of stiff ODE's is presented. These require only occasional calculation of the Jacobian matrix while maintaining stability. Especially, an effective second order stable algorithm with automatic stepsize control is designed and tested.
\end{abstract}

1. Introduction. During the last decade there has been a considerable amount of research on the numerical integration of stiff systems of ODE's. This work indicates that all efficient integration methods for such problems are implicit in character. This is due to the fact that only such methods have the required stability properties. Thus, the practical problem is not the stability restrictions, but the implicitness the need to avoid these give rise to. The relevant question is now, what is the cheapest type of implicitness we have to require.

Mainly, two different approaches to the implicitness can be found in the literature.

The first approach involves the numerical solution of nonlinear algebraic equations by the simplified Newton iteration. The simplification consists of treating the iteration matrix as piecewise constant (which means the use of an approximate Jacobian matrix). Examples of such an approach are semi-implicit Runge-Kutta formulas in N $\phi$ rsett [4] and the formulas based on backward-differences in Gear [3] .

Among recent methods proposed for numerical solution of stiff ODE's are the class of modified Rosenbrock methods introduced in Wolfbrandt [6]. When solving the system of equations

$$
y^{\prime}=f(y), \quad y\left(t_{0}\right)=y_{0},
$$

the formulas characterizing these methods are the following:

$$
\begin{aligned}
& y_{1}=y_{0}+h \sum_{i=1}^{\nu} b_{i} k_{i}, \\
& k_{i}=f\left(y_{0}+h \sum_{j=1}^{i-1} a_{i j} k_{j}\right)+h J_{0} \sum_{j=1}^{i} d_{i j} k_{j}, \quad i=1,2, \ldots, \nu,
\end{aligned}
$$

where $J_{0}$ denotes the Jacobian matrix $\partial f\left(y_{0}\right) / \partial y$.

Received February 22, 1978.

AMS (MOS) subject classifications (1970). Primary $65 \mathrm{L05}$. 
This is an example of the other approach to handle the implicitness. These methods maintain computational efficiency, since if such a method is applied to an $m$-dimensional system of stiff differential equations, then $\nu$ uncoupled systems of $m$ linear equations (with the same matrix if $d_{i i}=d$, all $i$ ) will only have to be solved at each integration step. However, they suffer from the practical disadvantage of computing the Jacobian $\partial f / \partial y$ at $y=y_{0}$.

A natural question is now, is it possible to replace $J_{0}$ in the formulas above with an arbitrary real square matrix $A$ (usually approximating $J_{0}$ )? An affirmative answer to this question will be given in this paper. In fact, the $W$-method introduced in the next section, combines the good things of the two approaches mentioned above.

2. The $W$-Methods. We consider numerical integration of system (1.1) using a class of methods of the form

$$
\begin{gathered}
y_{1}=y_{0}+h \sum_{i=1}^{\nu} b_{i} k_{i}, \\
W\left(h, d_{i i}, A\right) k_{i}=f\left(y_{0}+h \sum_{j=1}^{i-1} a_{i j} k_{j}\right)+h A \sum_{j=1}^{i-1} d_{i j} k_{j}, \quad i=1,2, \ldots, \nu,
\end{gathered}
$$

where $W\left(h, d_{i i}, A\right)=I-h d_{i i} A$, and $A$ is a real square matrix such that $W\left(h, d_{i i}, A\right)$ is invertible. The methods above will be called $\nu$-stage $W$-methods.

Remark. We note that if we choose $A \equiv 0$, or $A \equiv J_{0}$, then the $W$-methods reduce to the classical explicit Runge-Kutta methods and the modified Rosenbrock methods, respectively.

3. Order Conditions. In this section we will consider the order conditions for the $W$-methods.

We confine our attention first to the case $A \equiv 0$. Thus, the set of order conditions for a $W$-method includes those for a classical Runge-Kutta method. This demonstrates clearly the relation between these methods.

Next we turn to the case $A \equiv J_{0}$, corresponding to the modified Rosenbrock methods. The order conditions for these methods can originally be found in Wolfbrandt [6].

We combine these two sets of order conditions and further observe that the matrices $A$ and $J_{0}$ do not generally commutate. The order conditions for the $W$-methods are then readily obtainable. For illustration, the eight conditions for third order accuracy are given in Table I together with the associated 'elementary differentials' and their orders. To simplify these conditions, we have introduced the following notations:

$$
\begin{aligned}
M_{i} & =\sum_{j=1}^{i-1} a_{i j}, \quad i=2,3, \ldots, \nu \\
N_{i} & =\sum_{j=1}^{i} d_{i j}, \quad i=1,2, \ldots, \nu .
\end{aligned}
$$


TABLE I

Equations of conditions for a v-stage W-method

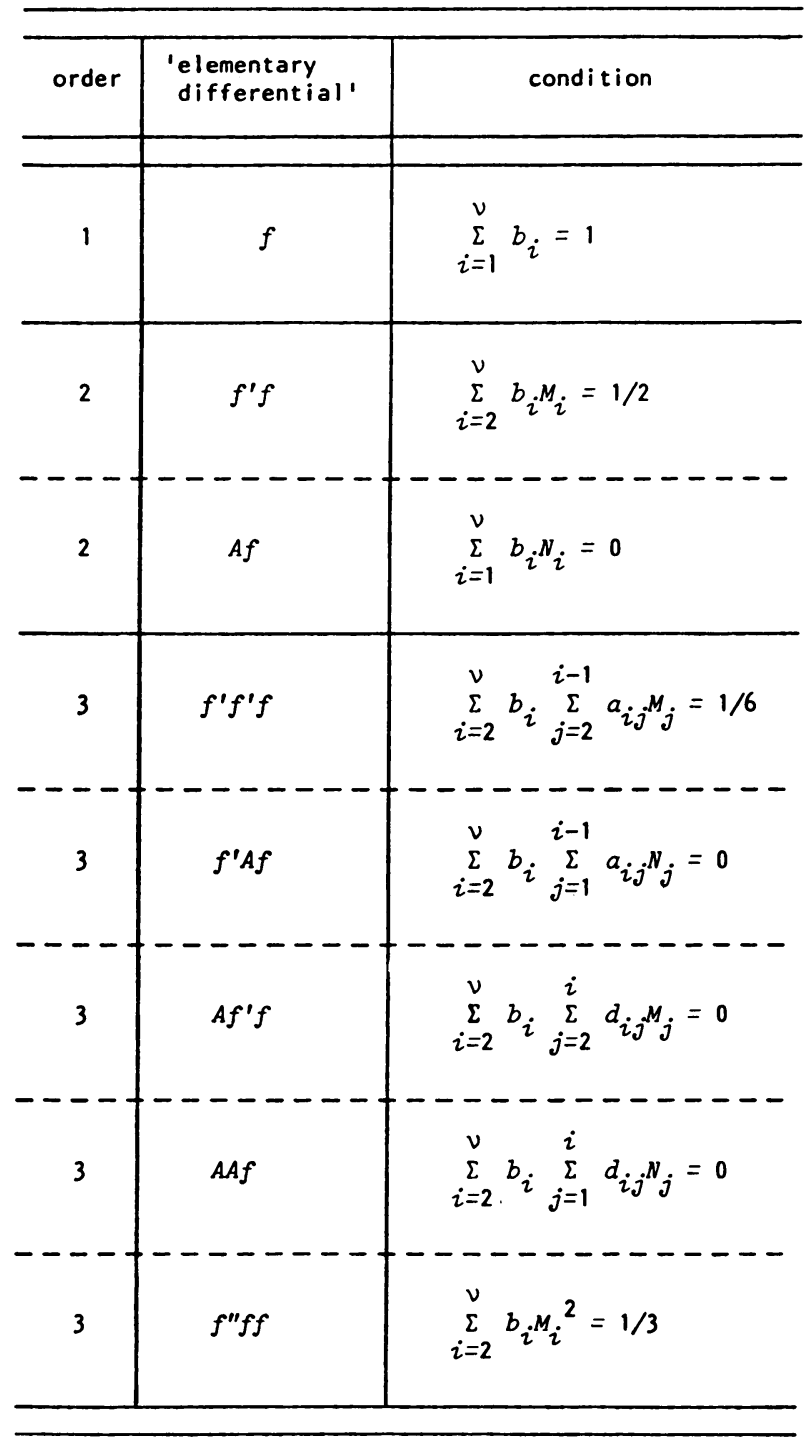

We shall now give an upper bound for the maximum order of a $W$-method. In preparation for this the following result in Wolfbrandt [7] is necessary. Let $\Pi_{r}=$ \{polynomials of degree $\leqslant r\}$ and

$$
R_{m}^{n}(u)=N_{n}(u) / D_{m}(u), \quad \text { where } N_{n} \in \Pi_{n} \text { and } D_{m} \in \Pi_{m} .
$$

LEMMA 1. The maximum attainable order of a rational approximation $R_{m}^{n}(u)$ to $\exp (u)$ with only real poles is $m+1$.

THEOREM 2. The maximum order for a $\nu$-stage $W$-method is at most $\nu+1$.

Proof. First, it is clear that a $\boldsymbol{W}$-method produces a rational approximation to $\exp (u)$ with only real poles when applied to the scalar equation $y^{\prime}=\lambda y$. 
Therefore, since the maximum order is attained when $A \equiv J_{0}$, the theorem follows from Lemma 1.

We recall from Section 1 that it is desirable from a practical point of view to choose $d_{i i}=d$, all $i$. The following surprising result, which is proved in Nørsett and Wolfbrandt [5], also shows that this choice is optimal for $A \equiv J_{0}$ in the sense of minimizing the absolute value of the error constant for a linear problem.

LEMMA 3. Let $R_{m}^{n}(u)$ be a rational approximation to $\exp (u)$ of order $m+1$ with only real poles, i.e.

$$
R_{m}^{n}(u)-\exp (u)=C u^{m+2}+O\left(u^{m+3}\right)
$$

Then the absolute value of the error constant $C$ attains its local minimum values when all the poles are equal.

The main conclusion of this discussion is that the 'best' we can do is choose $d_{i i}=d$, all $i$. This is also confirmed in Wolfbrandt [6] by computation of acceptability for the rational approximations mentioned in Lemma 3.

4. Explicit Formulas. Guided by the conclusions in Section 3 we will only consider $W$-methods with $d_{i i}=d$, all $i$. Moreover, we temporarily regard $d$ as fixed.

To match all terms up to second order for a 2-stage $W$-method, the coefficients of the formula (with one free parameter) must satisfy the following set of equations:

$$
b_{1}+b_{2}=1, \quad b_{2} a_{21}=1 / 2, \quad b_{2} d_{21}=-d .
$$

A natural way is to choose the free parameter, so that the method reduces to the improved Euler method when $A \equiv 0$, i.e.

$$
\begin{gathered}
y_{1}=y_{0}+\frac{h}{4}\left(k_{1}+3 k_{2}\right), \quad W(h, d, A) k_{1}=f\left(y_{0}\right), \\
W(h, d, A) k_{2}=f\left(y_{0}+\frac{2}{3} h k_{1}\right)-\frac{4}{3} h d A k_{1},
\end{gathered}
$$

where $W(h, d, A)=I-h d A$. The local truncation error $T$ for this method may be written as

$$
T=\frac{h^{3}}{3}\left\{f^{\prime} f^{\prime} f-3 d\left(f^{\prime} A f+A f^{\prime} f\right)+6 d^{2} A A f\right\}\left(y_{0}\right)+O\left(h^{4}\right) .
$$

We easily see that it is impossible to construct a third order 3-stage $W$-method. However, we can obtain a second order 3-stage formula with the local truncation error of the form

$$
T=\frac{1}{12} h^{3} f^{\prime} f^{\prime} f\left(y_{0}\right)+O\left(h^{4}\right)
$$

It is interesting to note that the matrix $A$ does not occur in the $h^{3}$-term.

For a 4-stage method, we can of course match up to and including $h^{3}$-terms. In fact, the parameters can be chosen so that the method only requires three function evaluations. 
Remark. We observe that a $\nu$-stage $W$-method with less than $\nu$ different function evaluations can be seen as a method with powers of $W(h, d, A)$ in its formula.

We also note that a 4 -stage $W$-method can be based on the classical fourth order Runge-Kutta method. In order to implement a $W$-method with variable stepsize it is necessary to compute the local truncation error. A device, proposed by England [1], allows us to estimate this error. The basic idea is to add extra stages to the method so that the new method is a more accurate one.

We consider the 2-stage $W$-method based on the improved Euler method. A third order estimate of the local truncation error is then of the form

$$
T=\frac{h}{8}\left(k_{1}-5 k_{2}+5 k_{3}-k_{4}\right)+O\left(h^{4}\right)
$$

where the extra stages $k_{3}$ and $k_{4}$ satisfy

$$
W(h, d, A) k_{3}=f\left(y_{1}\right), \quad W(h, d, A) k_{4}=f\left(y_{1}+\frac{2}{3} h k_{3}\right)+h d A\left(\frac{2}{3} k_{1}+6 k_{2}\right) .
$$

We adopt the notation $(2,4)-W$ for the above method with built-in error estimate. In a sequence of accepted integration steps of equal size this method will require only two function evaluations per step.

Our final task in this section is to extend $(2,4)-W$ to solve the nonautonomous system $y^{\prime}=f(y, t), y\left(t_{0}\right)=y_{0}$. We extend by appending $t_{0}+h a_{i}$ to the function evaluation in each stage $k_{i}, i=1,2,3$, and 4 . The corresponding set of order conditions in Table I has to be supplemented with the following 'nonautonomous' one

$$
\begin{aligned}
& \left(f_{t}\right) \quad b_{1} a_{1}+b_{2} a_{2}=1 / 2, \\
& \left(f_{t}\right) \quad c_{1} a_{1}+c_{2} a_{2}+c_{3} a_{3}+c_{4} a_{4}=1 / 2, \\
& \left(f_{t t}\right) \quad c_{1} a_{1}^{2}+c_{2} a_{2}^{2}+c_{3} a_{3}^{2}+c_{4} a_{4}^{2}=1 / 3, \\
& \left(f_{y} f_{t}\right) \quad c_{2} a_{21} a_{1}+c_{3}\left(a_{31} a_{1}+a_{32} a_{2}\right)+c_{4}\left(a_{41} a_{1}+a_{42} a_{2}+a_{43} a_{3}\right)=1 / 6, \\
& \left(A f_{t}\right) \quad c_{2} d_{21} a_{1}+c_{3}\left(d_{31} a_{1}+d_{32} a_{2}\right)+c_{4}\left(d_{41} a_{1}+d_{42} a_{2}+d_{43} a_{3}\right)=-d / 2, \\
& \left(f_{y t} f\right) \quad c_{2} M_{2} a_{2}+c_{3} M_{3} a_{3}+c_{4} M_{4} a_{4}=1 / 3,
\end{aligned}
$$

where the associated 'elementary differentials' are enclosed in parentheses. Thus, it is convenient to choose $a_{1}=0$ and $a_{i}=M_{i}, i=2,3$, and 4 .

5. Stability. $A(\alpha)$-stability, $L(\alpha)$-stability (or stiff stability) and its weaker associate strong stability, have become generally accepted as appropriate properties of numerical methods suitable for solving stiff ODE's.

Definition. A numerical method is said to be $A(\alpha)$-stable, $\alpha \in(0, \pi / 2)$, if all its solutions $\left\{y_{m}\right\}$ tend to zero, as $m \rightarrow \infty$, when the method is applied with fixed positive $h$ to the test equation 


$$
y^{\prime}=\lambda y, \quad \lambda \in S(\alpha),
$$

where $S(\alpha)=\{z \in C: z \neq 0,|\arg (-z)|<\alpha\}$.

A numerical method is said to be $A$-stable $(A(0)$-stable), if it is $A(\alpha)$-stable for all (some) $\alpha \in(0, \pi / 2) .^{*}$

Definition. An $A(\alpha)$-stable method, $\alpha \in(0, \pi / 2)$, is said to be strongly stable at infinity if all its solutions $\left\{y_{m}\right\}$, when the method is applied with fixed positive $h$ to the test equation (1.2), satisfy

$$
\lim _{|\lambda| \rightarrow \infty}\left|y_{m+1} / y_{m}\right| \leqslant c<1 \quad \text { when } \lambda \in S(\alpha) .
$$

If the real constant $c$ is equal to zero, then the method is called $L(\alpha)$-stable or stiffly stable. A numerical method is said to be $L$-stable $(L(0)$-stable), if it is $L(\alpha)$-stable for all (some) $\alpha \in(0, \pi / 2)$.

We shall now discuss stability properties of the $W$-methods. For simplicity, we assume that the matrices $A$ and $J_{0}$ commutate and that they are diagonalizable. Then the archetypical initial value problem is that in (1.2).

As mentioned before, a $W$-method results in a rational approximation to the exponential function with only real poles when applied to (1.2). Therefore, we first present in Table II some of the stability results for the modified Rosenbrock methods, i.e. the case $A \equiv J_{0}$, given in Wolfbrandt [6]. We have listed in this table acceptability angles and error constants for rational approximations.

$$
R_{\nu}^{\nu-1}(u) \equiv N_{\nu-1}(u) / D_{\nu}(u), \text { where } D_{\nu}(u)=(u-\gamma)^{\nu},
$$

to $\exp (u)$ of maximum order (which means that $\gamma$ is a zero to the Laguerre polynomial of degree $\nu$ and $d_{i i}=1 / \gamma$ ). The entries in the table represent the pair (acceptability angle; error constant) corresponding to $\gamma_{\nu}^{(k)}$, the $k$ th zero of the Laguerre polynomial of degree $\nu$ (the zeros are assumed to be ordered in increasing value).

TABLE II

L-acceptability angles and error constants

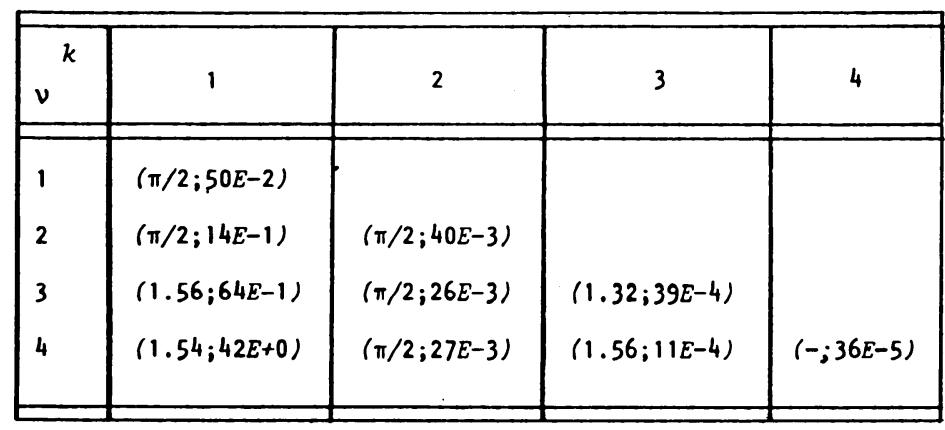

It would be nice to be able to derive the stability properties also for the case $A$ different from $J_{0}$. We shall content ourselves to discuss the $W$-method based on the improved Euler method.

${ }^{*} y_{m}$ is an approximation to $y\left(t_{m}\right)$, where $t_{m}=t_{0}+m h, m=0,1, \ldots$ 
We consider the scalar equation (1.2). Let the eigenvalue of $A$ be denoted by $a$. Then the following constraints for this method yield

$$
\begin{aligned}
& A(0) \text {-stable if and only if }|d a| \geqslant|\lambda| / 4-1 /(2 h), \\
& L(0) \text {-stable if and only if }|d a|=(1 \pm \sqrt{1 / 2})|\lambda| .
\end{aligned}
$$

It seems possible to handle these stability requirements by examining the local truncation error. Numerical experience shows that this works well for problems with transient to smooth components, while perhaps a more sophisticated control is necessary for problems with smooth to transient components.

6. A Comparison. The equations defining a semi-implicit Runge-Kutta method are

$$
\begin{aligned}
y_{1} & =y_{0}+h \sum_{i=1}^{\nu} b_{i} k_{i}, \\
k_{i} & =f\left(y_{0}+h \sum_{j=1}^{i-1} a_{i j} k_{j}+h d_{i i} k_{i}\right), \quad i=1,2, \ldots, \nu .
\end{aligned}
$$

This method, as well as a $W$-method with $A \equiv J_{0}$, produces a rational approximation to $\exp (h \lambda)$ with only real poles when applied to the test equation $y^{\prime}=\lambda y$. In view of this relation a brief comparison between these methods will be done in this section.

The semi-implicit Runge-Kutta methods are implicit in character involving solution of $\nu$ uncoupled nonlinear equations of the form

$$
F\left(k_{i}\right) \equiv k_{i}-f\left(y_{0}+h \sum_{j=1}^{i-1} a_{i j} k_{j}+h d_{i i} k_{i}\right)=0, \quad i=1,2, \ldots, \nu,
$$

by the simplified Newton iteration

$$
\begin{array}{r}
W\left(h, d_{i i}, J_{0}\right) k_{i}^{(m)}=f\left(y_{0}+h \sum_{j=1}^{i-1} a_{i j} k_{j}^{\left(M_{j}\right)}+h d_{i i} k_{i}^{(m-1)}\right)-h d_{i i} J_{0} k_{i}^{(m-1)}, \\
m=1,2, \ldots, M_{i} \text { and } i=1,2, \ldots, \nu,
\end{array}
$$

where $k_{i}^{(0)}$ is some suitable starting value and $M_{i}$ is the maximum number of iterations required.

It is a common practice to employ a linear combination of known iterated values to provide $k_{i}^{(0)}$ which we write as

$$
k_{i}^{(0)}=\sum_{j=1}^{i-1} \frac{d_{i j}}{d_{i i}} k_{j}^{\left(M_{j}\right)} .
$$

Thus, the modified Rosenbrock method in Wolfbrandt [6] can be regarded as a linearization of a semi-implicit Runge-Kutta method with the above choice of $k_{i}^{(0)}$. Moreover, replacing $J_{0}$ by $A$ in the iteration formula above we obtain a $W$-method.

The traditional motivation behind the choice of the parameters in the expression for $k_{i}^{(0)}$ is based on obtaining a good initial approximation to $k_{i}$. A somewhat more attractive approach, in our opinion, is to avoid the necessity to iterate. This will be illustrated in the following example. 
Example. We consider the generalized midpoint-rule, alias the $\theta$-method

$$
y_{1}=y_{0}+h k, \quad k=f\left(y_{0}+h \theta k\right) .
$$

After two iterations it yields

$$
W\left(h, \theta, J_{0}\right) k_{1}=f\left(y_{0}\right), \quad W\left(h, \theta, J_{0}\right) k_{2}=f\left(y_{0}+h \theta k_{1}\right)-h \theta J_{0} k_{1},
$$

where $k_{m} \equiv k^{(m)}, m=1$ and $2\left(k_{0}^{(0)} \equiv 0\right)$. Putting $\widetilde{y}_{1}=y_{0}+h k_{2}$, we obtain a 2 stage $W$-method.

We recall from Section 3 that the order of $\widetilde{y}_{1}$ is less than 2 for $\theta \neq 1 / 2$, otherwise 2.

First, we will assume that an exact Jacobian matrix $J_{0}$ is used in the simplified Newton iteration. Then there is no gain in stability to iterate. Moreover, numerical experiments indicate that only a small number of iterations are required in the $\theta$-method. We easily verify that the dominating local truncation error terms for this method are independent of the number of iterations. In fact, the local truncation error can be expressed as

$$
T=h^{2}\left(\theta-\frac{1}{2}\right) f^{\prime} f\left(y_{0}\right)+h^{3}\left\{\left(\theta^{2}-\frac{1}{3}\right) f^{\prime \prime} f f+\left(\theta^{2}-\frac{1}{6}\right) f^{\prime} f^{\prime} f\right\}\left(y_{0}\right)+O\left(h^{4}\right) .
$$

This shows again that it becomes no great benefit in making several iterations when an exact Jacobian matrix $J_{0}$ is used.

We observe that the $W$-method based on the improved Euler method has a local truncation error of the form

$$
T=h^{3}\left(\frac{1}{6}-d+d^{2}\right) f^{\prime} f^{\prime} f\left(y_{0}\right)+O\left(h^{4}\right) \quad \text { when } A \equiv J_{0} .
$$

In the asymptotic region many stiff systems behave as linear systems. Therefore, an approximation to the Jacobian matrix $J_{0}$ in the simplified Newton iteration can be used for a large number of integration steps. Accordingly, we next assume that $J_{0}$ is replaced by an approximation $\boldsymbol{A}$ and consider the effect of iterations in the $\theta$-method. As noted previously, this method with $M$ iterations may be regarded as an $M$-stage $W$ method with $a_{i j}=-d_{i j}=\theta$, for $j=i-1, i=1,2, \ldots, M$ and $b_{i}=1$ for $i=M$, otherwise zero. This method will in the following be called the degenerated $W$-method.

In contrast to the case of exact Jacobian the dominating local truncation error constants are now dependent of the first two iterations, but only of these. The error constants together with the associated 'elementary differentials' and the number of iterations are listed in Table III below.

From this important result we conclude that iteration is one way to eliminate the influence on accuracy of an approximate Jacobian matrix. However, the special 3-stage $W$-method in Section 4 has order 2 for all $d$ and share the above-mentioned properties of the degenerated 3-stage method too.

The changes due to iterations of the $A(0)$-stability contour for the $\theta$-method is illustrated in Figures 6.1-6.2 for $\theta=1 / 2$ (maximum order) and $\theta=1$ ( $L$-stability), respectively. In these figures the convergence constraint is also drawn. To 
interpret the figures we note that the stability regions are to the left of the contours.

TABLE III

Error constants

\begin{tabular}{r|r|r|r|r|r|r|r}
\hline$m$ & \multicolumn{1}{|c|}{$f^{\prime} f$} & $A f$ & $f^{\prime} f^{\prime} f$ & $A f^{\prime} f$ & $f^{\prime} A f$ & $A A f$ & $f^{\prime \prime} f f$ \\
\hline 1 & $-1 / 2$ & $\theta$ & $-1 / 6$ & 0 & 0 & $\theta^{2}$ & $\theta^{2}-1 / 3$ \\
2 & $\theta-1 / 2$ & 0 & $-1 / 6$ & $\theta^{2}$ & $\theta^{2}$ & $-\theta^{2}$ & $\theta^{2}-1 / 3$ \\
3 & $\theta-1 / 2$ & 0 & $\theta^{2}-1 / 6$ & 0 & 0 & 0 & $\theta^{2}-1 / 3$ \\
- & - & - & - &. & - & $\cdot$ & $\cdot$ \\
$\infty$ & $\theta-1 / 2$ & 0 & $\theta^{2}-1 / 6$ & 0 & 0 & 0 & $\theta^{2}-1 / 3$ \\
\hline
\end{tabular}

From these figures we conclude that 'convergence' is stronger (weaker) than 'stability' for $\theta=1(1 / 2)$. Further, the 'stability' region for the 2 -stage $W$-method based on the improved Euler method contains the regions in the figures for $d \geqslant 1 / 2$.

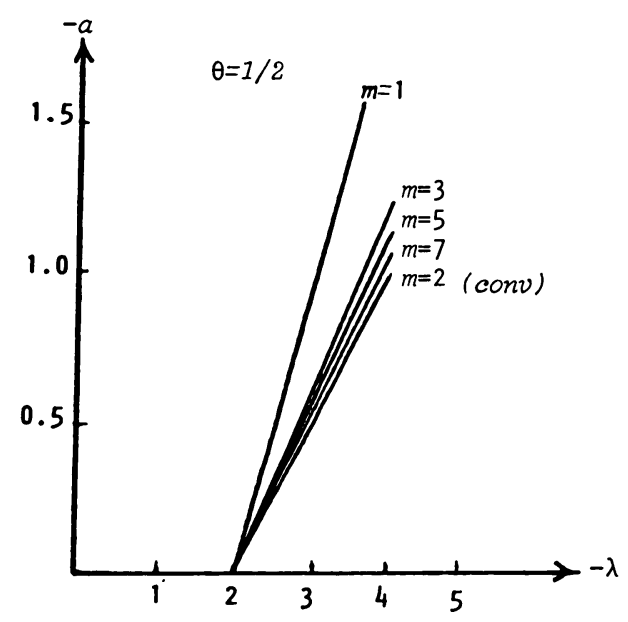

Figure 6.1

$A(0)$-stability contour

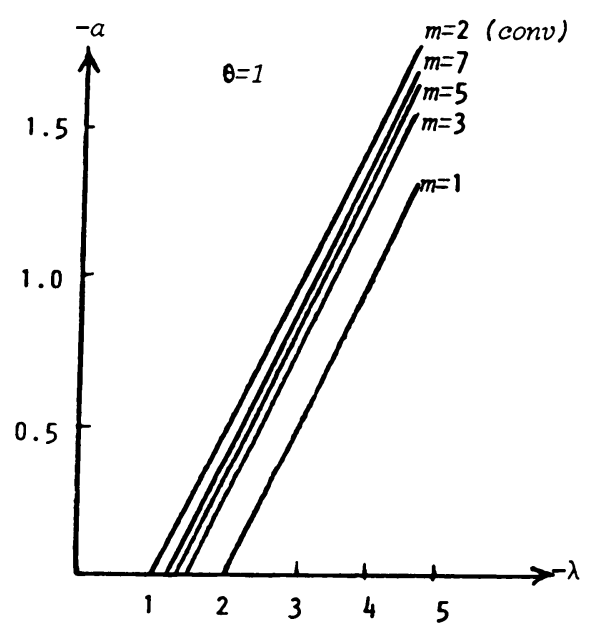

FIGURE 6.2 A(0)-stability contour

Finally, for the $\theta$-method with $\theta=1$ it is possible to iterate with an approximate Jacobian and yet obtain $L$-stability, while this is not the case for a $W$-method.

7. Numerical Examples. The intention of this section is to present an algorithm for solving stiff ODE's. The underlying method is the $(2,4)-W$-method with $d=1-$ $\sqrt{1 / 2}$, i.e. $d=1 / \gamma_{2}^{(2)}$ (see Table II). The design of such an algorithm is a very complex work involving numerous choices in equation solver, error estimation and stepsize control as well as features of detailed programming. We have not attempted to construct a complete algorithm. However, we have concentrated on some of the difficulties associated with the stepsize control and the reevaluation of the matrix $A$. 
The linear systems in the method have the form

$$
W(h, d, A) k=f, \quad \text { where } W(h, d, A) \equiv I-d h A .
$$

These are solved by Gaussian elimination, i.e. $L U$-decomposition and back-substitution. Therefore, during the computation the $W$-matrix is kept as long as possible and is reevaluated only after a failure of a specified accuracy requirement for the local truncation error.

The purpose of the control of stepsize is to integrate as efficiently as possible keeping the error under a prescribed level. The optimal stepsize is not available as it depends on the entire solution. Therefore, the stepsize is based on the local truncation error. The algorithm tests that the error-per-step is less than a tolerance requested. The strategy for optimal stepsize is calculated as shown below.

We denote the norm of the error estimate with current stepsize $h$ by $E(h)$. Then the optimal stepsize is $p h, p>0$, satisfying $E(p h)=\tau$, where $\tau$ is the error tolerance. Since the method is of order 2 , then $p=(\tau / E(h))^{1 / 3}$. This strategy is critical to the efficiency, since changes in stepsize will lead to extra $L U$-decompositions, Jacobian evaluations and function evaluations. Therefore, an increase in the stepsize should only be attempted unless $p \geqslant q>1$. Also, when changing stepsize upward or downward, the new value would be set at $r h$, for some $r<p$. Finally, before increasing the stepsize it is very economical to retain the old value one step further without making any error estimation.

The strategy we have adopted is based on a combination of heuristics and numerical experiments. The matrix $W$ is recalculated only when the stepsize is changed or when the matrix $A$ is altered and set equal to the Jacobian matrix. The latter happens only when $E(h)>0.7 \tau$ and $A \neq J_{0}$. The table below gives the appropriate actions.

TABLE IV

Values of the parameter $r$

\begin{tabular}{|c|c|c|}
\hline Error & $A \bar{\equiv} J_{0}$ & $A \not J_{0}$ \\
\hline$E(h)<\tau$ & $\begin{array}{l}\max \{1,0.9[p]\} \\
\text { Accepted step }\end{array}$ & $\begin{array}{l}\{1,0.9[p]\} \\
\text { Accepted step }\end{array}$ \\
\hline$\tau \leq E(h)<2 \tau$ & $\begin{array}{l}0.85 p \\
\text { Rejected step }\end{array}$ & $\begin{array}{l}1 \\
\text { Accepted step }\end{array}$ \\
\hline$E(h) \geq 2 \tau$ & $\begin{array}{l}0.85 p \\
\text { Rejected step }\end{array}$ & $\begin{array}{l}0.80 p \\
\text { Rejected step }\end{array}$ \\
\hline
\end{tabular}

In order to avoid rejection of steps and for stability reasons we have also found it convenient to change the stepsize to $0.9 p h$ when $0.8 \tau \leqslant E(h) \leqslant 2 \tau$ in the first step with an 'old' Jacobian matrix. Numerical results for the $(2,4)-W$-method on four problems are given below. For comparison we have also included results from Nørsett [4], Enright, Hull and Lindberg [2]. 


\section{Problem 1.}

$$
\begin{aligned}
& y_{1}^{\prime}=0.01-\left(1+\left(y_{1}+1000\right)\left(y_{1}+1\right)\right)\left(0.01+y_{1}+y_{2}\right), \quad y_{1}(0)=0 \\
& y_{2}^{\prime}=0.01-\left(1+y_{2}^{2}\right)\left(0.01+y_{1}+y_{2}\right), \quad y_{2}(0)=0, \quad t \in[0,100]
\end{aligned}
$$

A relative error measure based on a weighted Euclidean norm is used with a vector of weights made up of the numerically greatest solution points so far encountered for each component.

\section{TABLE V}

Number of function evaluations, Jacobian evaluations and steps for Problem 1

\begin{tabular}{c||l|l|l|}
$\tau$ & EhIE1A3 & GEAR & IRKSUB4 \\
\hline $1 E-3$ & $892 / 50 / 66$ & $181 / 20 / 69$ & $185 / 12 / 30$ \\
\hline $1 E-5$ & $1908 / 97 / 144$ & $380 / 36 / 136$ & $367 / 25 / 54$ \\
\hline
\end{tabular}

\begin{tabular}{c|l|l|}
$T$ & SIRSPN & $(2,4)-W$ \\
\hline $1 E-3$ & $250 / 40 /-$ & $92 / 14 / 45$ \\
\hline $1 E-5$ & $568 / 39 /-$ & $378 / 34 / 181$ \\
\hline
\end{tabular}

Problem 2.

$$
y^{\prime}=U B U y+U w, \quad y(0)=[0,-2,-1,-1]^{T},
$$

where

$$
B=\left|\begin{array}{rrrc}
-10^{4} & 10^{4} & 0 & 0 \\
-10^{4} & -10^{4} & 0 & 0 \\
0 & 0 & -1 & 0 \\
0 & 0 & 0 & -10^{-3}
\end{array}\right| \text {, }
$$

$U$ is the unitary matrix with diagonal elements equal to $-1 / 2$ and all other elements equal to $1 / 2$,

$$
w=\left[\left(z_{1}^{2}-z_{2}^{2}\right) / 2, z_{1} z_{2}, z_{3}^{2}, z_{4}^{2}\right]^{T}, \quad z=U y, \quad t \in[0,100] .
$$

The same relative error measure as in Problem 1 is used. 
TABLE VI

Number of function evaluations and Jacobian evaluations for Problem 2

\begin{tabular}{c||c|c|c|c|c|}
$\tau$ & \multicolumn{1}{|c}{ GEAR } & IMPEX2 & IRKSUB4 & SIRSPN & $(2,4)-W$ \\
\hline $1 E-3$ & $581 / 40$ & $548 / 22$ & $479 / 23$ & $334 / 26$ & $190 / 29$ \\
\hline $1 E-5$ & $959 / 45$ & $1032 / 24$ & $1095 / 34$ & $1202 / 27$ & $694 / 33$ \\
\hline
\end{tabular}

Problem 3.

$$
\begin{array}{ll}
y_{1}^{\prime}=-0.04 y_{1}+0.01 y_{2} y_{3}, & y_{1}(0)=1, \\
y_{2}^{\prime}=400 y_{1}-100 y_{2} y_{3}-3000 y_{2}^{2}, & y_{2}(0)=0, \\
y_{3}^{\prime}=30 y_{2}^{2}, & y_{3}(0)=0, \quad t \in[0,40] .
\end{array}
$$

For this problem the error tolerance $\tau \equiv \tau(t)$ is defined by $\tau(t)=6 h \epsilon /\left(t-t_{0}\right)$, where $\epsilon$ is the global tolerance and $t-t_{0}$ is the distance from the initial point to the end of the current step (see Enright et al. [2]). Moreover, the maximum norm is used.

\section{TABLE VII}

Number of function evaluations, Jacobian evaluations, LU-decompositions, and steps for Problem 3

\begin{tabular}{c||l|l|l|}
$\varepsilon$ & GEAR & SDBASIC & TRAPEX \\
\hline $1 E-2$ & $131 / 14 / 14 / 55$ & $241 / 176 / 92 / 28$ & $237 / 29 / 29 / 11$ \\
\hline
\end{tabular}

\begin{tabular}{c||l|l|l|}
$\varepsilon$ & GENRK & IMPRK & $(2,4)-W$ \\
\hline $1 E-2$ & $99 / 9 / 27 / 9$ & $387 / 89 / 89 / 11$ & $91 / 15 / 15 / 41$ \\
\hline
\end{tabular}

Problem 4. This arises from solving parabolic partial differential equations. Consider the parabolic equation

$$
\frac{\partial u}{\partial t}-\frac{\partial}{\partial x}\left(a\left(\frac{\partial u}{\partial x}\right) \frac{\partial u}{\partial x}\right)=0
$$

in the region $[0,1] \times[0, \infty]$ subject to the initial condition $u(x, 0)=u_{0}(x), x \in$ $(0,1)$ and the boundary condition $u(0, t)=u(1, t)=0, t \geqslant 0$. Let $a(z)=\nu z^{2}+1$, $\nu \geqslant 0$ and $u_{0} \equiv 1$. Finite element approximation in the space variable $x$ results in the semidiscrete Galerkin approximation, which is a stiff system of $N$ ODE's of the form 


$$
B \frac{d U}{d t}+K(U) U=0, \quad t>0, \quad U(0)=U_{0},
$$

where $B$ and $K$ are the mass- and stiffness-finite element matrices, respectively. Here we use linear splines with equidistant knots. An appropriate norm is the weighted Euclidean norm with all weights equal to $\Delta x$, where $\Delta x=1 /(N+1)$.

Numerical results for the $(2,4)-W$-method on this problem are shown in Table VIII and Table IX for $\nu=0$ (which corresponds to a linear problem) and $\nu=1$, respectively. For illustration we have presented the numerical solution in the following adequate points $(x, t): x=0.1,0.3$ and $t=0.01,1,0$.

Remark. The number of $L U$-decompositions and the number of Jacobian evaluations is nearly identical for the $(2,4)-W$-method on the problems given. In addition, no extra function evaluations are spent on the stepsize control. The only price to be paid for the stepsize control is the cost of one back-substitution.

8. Conclusions. The aim was to introduce a new class of linear implicit methods without exact Jacobian for solving stiff ODE's. We do not claim to have found an ideal algorithm for such problems, but the theoretical and numerical results achieved for the introduced $(2,4)$ - $W$-method merit a further investigation of higher order $W$-methods.

TABLE VIII

Results for (2, 4)-W on Problem 4 with $v=0$

\begin{tabular}{|c|c|c|c|c|c|}
\hline \multicolumn{6}{|c|}{ Numerical Solution } \\
\hline \multirow[b]{2}{*}{$\Delta x$} & \multirow[b]{2}{*}{$\tau$} & \multicolumn{2}{|c|}{$t=0.01$} & \multicolumn{2}{|c|}{$t=1.0$} \\
\hline & & $x=0.1$ & $x=0.3$ & $x=0.1$ & $x=0.3$ \\
\hline $1 / 10$ & $1 E-3$ & 0.4910 & 0.9646 & $0.1141 E-4$ & $0.2991 E-4$ \\
\hline $1 / 20$ & $\begin{array}{l}1 E-3 \\
1 E-5\end{array}$ & $\begin{array}{l}0.5100 \\
0.5101\end{array}$ & $\begin{array}{l}0.9656 \\
0.9653\end{array}$ & $\begin{array}{l}0.1364 E-4 \\
0.2163 E-4\end{array}$ & $\begin{array}{l}0.3570 E-4 \\
0.5664 E-4\end{array}$ \\
\hline $1 / 40$ & $\begin{array}{l}1 E-5 \\
1 E-6\end{array}$ & $\begin{array}{l}0.5167 \\
0.5185\end{array}$ & $\begin{array}{l}0.9655 \\
0.9662\end{array}$ & $\begin{array}{l}0.2237 E-4 \\
0.2126 E-4\end{array}$ & $\begin{array}{l}0.5857 E-4 \\
0.5565 E-4\end{array}$ \\
\hline \multicolumn{6}{|c|}{ Exact Solution } \\
\hline & & 0.5187 & 0.9669 & $0.2035 E-4$ & $0.5328 E-4$ \\
\hline \multicolumn{6}{|c|}{ Number of Function Evaluations, Jacobian Evaluation and Steps } \\
\hline$\Delta x$ & $\tau$ & & & & \\
\hline $1 / 10$ & $1 E-3$ & $18 / 3 / 8$ & & $61 / 10 / 30$ & \\
\hline $1 / 20$ & $\begin{array}{l}1 E-3 \\
1 E-5\end{array}$ & $\begin{array}{l}25 / 4 / 1 \\
100 / 5 /\end{array}$ & & $\begin{array}{l}71 / 11 / 25 \\
280 / 14 / 139\end{array}$ & \\
\hline $1 / 40$ & $\begin{array}{l}1 E-5 \\
1 E-6\end{array}$ & $\begin{array}{l}136 / 8 / \\
282 / 8 /\end{array}$ & & $\begin{array}{l}318 / 17 / 158 \\
658 / 17 / 328\end{array}$ & \\
\hline
\end{tabular}




\section{TABLE IX}

Results for $(2,4)-W$ on Problem 4 with $v=1$

\begin{tabular}{|c|c|c|c|c|c|}
\hline \multicolumn{6}{|c|}{ Nhonerical Solution } \\
\hline \multirow[b]{2}{*}{$\Delta x$} & \multirow[b]{2}{*}{$\tau$} & \multicolumn{2}{|c|}{$t=0.01$} & \multicolumn{2}{|c|}{$t=1.0$} \\
\hline & & $x=0.1$ & $x=0.3$ & $x=0.1$ & $x=0.3$ \\
\hline $1 / 10$ & $1 E-3$ & 0.3023 & 0.9508 & $0.6531 E-5$ & $0.1662 E-4$ \\
\hline $1 / 20$ & $\begin{array}{l}1 E-3 \\
1 E-5\end{array}$ & $\begin{array}{l}0.3034 \\
0.3020\end{array}$ & $\begin{array}{l}0.8066 \\
0.8041\end{array}$ & $\begin{array}{l}0.4835 E-5 \\
0.1503 E-4\end{array}$ & $\begin{array}{l}0.1267 E-4 \\
0.3935 E-4\end{array}$ \\
\hline $1 / 40$ & $\begin{array}{l}1 E-5 \\
1 E-6\end{array}$ & $\begin{array}{l}0.3023 \\
0.3026\end{array}$ & $\begin{array}{l}0.8041 \\
0.8045\end{array}$ & $\begin{array}{l}0.1344 E-4 \\
0.1266 E-4\end{array}$ & $\begin{array}{l}0.3518 E-4 \\
0.3315 E-4\end{array}$ \\
\hline \multicolumn{6}{|c|}{ Nromber of Function Evaluations, Jacobian evaluations and Steps } \\
\hline$\Delta x$ & $\tau$ & \multicolumn{2}{|c|}{$t=0.01$} & \multicolumn{2}{|l|}{$t=1.0$} \\
\hline $1 / 10$ & $1 E-3$ & \multicolumn{2}{|c|}{$38 / 6 / 18$} & \multicolumn{2}{|l|}{$85 / 14 / 42$} \\
\hline $1 / 20$ & $\begin{array}{l}1 E-3 \\
1 E-5\end{array}$ & \multicolumn{2}{|c|}{$\begin{array}{l}56 / 10 / 27 \\
264 / 14 / 130\end{array}$} & \multicolumn{2}{|c|}{$\begin{array}{l}99 / 17 / 49 \\
476 / 23 / 236\end{array}$} \\
\hline $1 / 40$ & $\begin{array}{l}1 E-5 \\
1 E-6\end{array}$ & \multicolumn{2}{|c|}{$\begin{array}{l}380 / 20 / 186 \\
851 / 17 / 423\end{array}$} & \multicolumn{2}{|c|}{$\begin{array}{l}610 / 29 / 301 \\
1309 / 27 / 652\end{array}$} \\
\hline
\end{tabular}

School of Organization and Management

Yale University

Box 1 A

New Haven, Connecticut 06520

Department of Computer Sciences

Chalmers University of Technology

Fack

S-402 20 Goteborg, Sweden

1. R. ENGLAND, "Error estimates for Runge-Kutta type solutions to systems of ordinary differential equations," Comput J., v. 12, 1969, pp. 166-170.

2. W. H. ENRIGHT, T. E. HULL \& B. LINDBERG, "Comparing numerical methods for stiff systems of o.d.e.:s," $B I T, \mathrm{v} .15,1975$, pp. 1-10.

3. C. W. GEAR, Numerical Initial Value Problems in Ordinary Differential Equations, Prentice-Hall, Englewood Cliffs, N. J., 1971.

4. S. P. NQRSETT, Semi-Explicit Runge-Kutta Methods, Technical Report 6, Dept. of Math., Univ. of Trondheim, 1974.

5. S. P. NØRSETT \& A. WOLFBRANDT, "Attainable order of rational approximations to the exponential function with only real poles," $B I T$, v. 17, 1977, pp. 200-208.

6. A. WOLFBRANDT, A Study of Rosenbrock Processes with Respect to Order Conditions and Stiff Stability, Thesis, Chalmers Univ. of Technology, Goteborg, Sweden, 1977.

7. A. WOLFBRANDT, "A note on a recent result of rational approximation to the exponential function," $B I T$, v. 17, 1977, pp. 367-368. 